\title{
Attentional control in the attentional blink is modulated by odor
}

\author{
Lorenza S. Colzato • Roberta Sellaro • \\ Claudia Rossi Paccani • Bernhard Hommel
}

Published online: 29 July 2014

(C) The Psychonomic Society, Inc. 2014

\begin{abstract}
Increasing evidence suggests that 'aromas have distinctive effects on the allocation of attention in space: Arousing olfactory fragrances (e.g., peppermint) are supposed to induce a more focused state, and calming olfactory fragrances (e.g., lavender) a broader attentional state. Here, we investigate whether odors have similar effects on the allocation of attention in time. Participants performed the attentional blink $(\mathrm{AB})$ task, known to produce a deficit in reporting the second of two target stimuli presented in close succession in a rapid sequence of distractors, while being exposed to either a peppermint or a lavender aroma. In two experiments using a between-subjects and a within-subjects design, respectively, we show that the two odors have specific effects on attentional control: As compared with the calming lavender aroma, the arousing peppermint condition yielded a larger AB. Our results demonstrate that attentional control is systematically modulated by factors that induce a more or a less distributed state of mind.
\end{abstract}

Keywords Attentional blink - Aromas - Attentional state

\section{Introduction}

Odors have a power of persuasion stronger than that of words, appearances, emotions, or will. The persuasive power of an odor cannot be fended off, it enters into us

L. S. Colzato · R. Sellaro · C. Rossi Paccani • B. Hommel Institute for Psychological Research \& Leiden Institute for Brain and Cognition, Leiden University, Leiden, The Netherlands

L. S. Colzato $(\square)$

Cognitive Psychology Unit, Leiden University, Wassenaarseweg 52,

2333 AK Leiden, The Netherlands

e-mail: colzato@fsw.leidenuniv.nl like breath into our lungs, it fills us up, imbues us totally.

There is no remedy for it.

\section{Patrick Süskind, Perfume: The Story of a Murderer}

Aromas have been found to bias differentially individual's attention toward either global or local representational levels (for reviews, see Hertz, 2009; Johnson, 2011). The stimulating aroma peppermint is supposed to increase arousal and induce a more focused attentional state, while lavender, a calming olfactory fragrance, is considered to induce relaxation and a broader attentional state (Barker et al., 2003; Basevitch et al., 2011; Ho \& Spence, 2005; Ilmberger et al., 2001; Moss, Cook, Wesnes, \& Duckett, 2003). Previous studies have looked into the allocation of attention in space, assuming (and showing) that a more focused or distributed state is associated with a smaller and broader spatial focus of attention, respectively (Dale \& Arnell, 2010). In this study, we investigated the possibility that odors (peppermint and lavender aromas) affect the allocation of attention in time.

A widely accepted tool for assessing the allocation of attention over time is the so-called attentional blink (AB) task (Raymond, Shapiro, \& Arnell, 1992). The AB occurs if two masked (or otherwise difficult to identify) target stimuli appear in close temporal proximity, such as in tasks using rapid serial visual presentation (RSVP): While the first target (T1) is typically easy to report accurately, the identification of the second target (T2) is drastically impaired if it follows $\mathrm{T} 1$ within $100-500 \mathrm{~ms}$. It is widely assumed that the $\mathrm{AB}$ reflects a processing bottleneck: Reporting a stimulus is assumed to require transferring its sensory representation to and consolidating it in working memory-a process that is supposed to draw on attentional resources that are occupied for processing $\mathrm{T} 1$ and, thus, temporarily unavailable for T2 processing (Chun \& Potter, 1995; Jolicoeur \& Dell'Acqua, 1998; Vogel, Luck, \& Shapiro, 1998; for a recent review, see Dux \& Marois, 2009). 
However, even though the $\mathrm{AB}$ is a robust phenomenon, there is mounting evidence for the role of strategic, intra- and interindividual differences in modulating the severity of the $\mathrm{AB}$. For instance, performance on $\mathrm{T} 2$ increases, sometimes even dramatically, when $\mathrm{T} 1$ and $\mathrm{T} 2$ are presented in a longer sequence of multiple targets (Di Lollo, Kawahara, Ghorashi, \& Enns, 2005) and if participants are assuming a relaxed attitude toward the task (Olivers \& Nieuwenhuis, 2005). ${ }^{1}$ These results suggest that individuals and/or environmental conditions can exert control over the allocation of attention when processing targets and that the allocation policy affects the size of the $\mathrm{AB}$.

Indeed, electrophysiological and magnetoencephalographic markers of attentional processes were found to be very sensitive to experimental manipulations of participants' expectations regarding the timing and probability of target presentations in $\mathrm{AB}$ tasks (Akyürek, Riddell, Toffanin, \& Hommel, 2007; Gross et al., 2006; Martens \& Johnson, 2005). In addition, Shapiro, Schmitz, Martens, Hommel, and Schnitzler (2006) provided evidence that the individual size of the $A B$ is predicted by the amount of attentional resources devoted to $\mathrm{T} 1$ processing (as indicated by the individual amplitude of the M3, the magnetoencephalographic equivalent of the electrophysiological P3). Interestingly, even though more resources were allocated to T1, T1 accuracy did not improve, suggesting that humans may overinvest attentional resources into T1 processing - in turn, impairing T2 performance (Olivers \& Nieuwenhuis, 2005, 2006; Taatgen, Juvina, Schipper, Borst, $\&$ Martens, 2009). This overinvestment hypothesis is consistent with findings from other studies on individual differences, which consistently show effects on $\mathrm{T} 2$ but not $\mathrm{T} 1$ report (e.g., Colzato et al., 2008; Colzato, Spapè, Pannebakker, \& Hommel, 2007; Colzato, Slagter, Spapè, \& Hommel, 2008; Colzato et al., 2011; Dale \& Arnell, 2010; Martens, Munneke, Smid, \& Johnson, 2006; Martens \& Valchev, 2009).

The overinvestment hypothesis suggests that focusing too much on the task is likely to be counterproductive in a multiple-target RSVP condition, since investing a morethan-necessary amount of attentional resources to the processing of each target might leave too few resources for the next upcoming target — especially if the time between the targets is short. This suggests that aromas that induce more focusing of attention might lead to a more pronounced $\mathrm{AB}$ than aromas that are more relaxing: Being exposed to peppermint aroma should be more likely to induce attentional overinvestment and lead to larger $\mathrm{AB}$ than being exposed to lavender. We used

\footnotetext{
${ }^{1}$ Note that the general beneficial effect of reducing the available attentional resources (e.g., by distraction through an additional task) on $\mathrm{AB}$ performance has been replicated several times by now, including Olivers and Nieuwenhuis's own 2005 and 2006 articles. However, the effect of music (Olivers \& Nieuwenhuis, 2005) has been failed to replicate by T. Spalek and V. Di Lollo using the identical musical tune (personal communication).
}

a between-group (Experiment 1) and a within-subjects (Experiment 2) design to test this hypothesis. In both experiments, participants performed RSVP tasks in a peppermintscented and/or a lavender-scented room, and we expected that peppermint (due to its arousing effect and the focused attentional state it induces) would lead to a more pronounced $\mathrm{AB}$ than the less arousing lavender aroma.

\section{General method}

Apparatus and stimuli

Both experiments were controlled by a Targa Pentium III computer. All stimuli were presented in a resolution of $800 \times 600$ pixels in 16-bit color on a 17-in. CRT refreshing at $100 \mathrm{~Hz}$. Participants were seated at a viewing distance of about $50 \mathrm{~cm}$. The fixation mark (“+”) and all RSVP items were presented centrally in black on a gray background (RGB $128,128,128)$. Each item was set in 16-point Times New Roman font. RSVP items included letters and digits. Letters were drawn randomly without replacement from the alphabet. Digits were drawn randomly from the set 2-9.

\section{RSVP task}

In the RSVP task adopted from Colzato, Slagter, de Rover, and Hommel (2011), participants had to identify and report two digits ( $\mathrm{T} 1$ and $\mathrm{T} 2$ ) presented in a rapid stream of letter distractors. Given the sensitivity of the RVSP to verbal instructions, all participants received the same standard written (Dutch) instructions on the computer screen, saying the following: "You will see a series of letters and numbers rapidly appearing on the computer screen. Your task is to remember the digits in the series. When the series ends, you have to respond to the question 'which two digits did you see?' You type in the digits you saw (order does not matter), and then another series will appear." After reading the instructions, which included a slow demonstration of the RSVP stream, and a confirmation that the participant to had fully understood the task, participants underwent 24 trials of training. If more than $50 \%$ of the responses were incorrect during the training, the training part was automatically repeated. A fixation "plus" sign, which was shown for 2,000 ms, marked the beginning of each trial. After a blank interval of $250 \mathrm{~ms}$, the RSVP commenced, consisting of 20 items with a duration of $70 \mathrm{~ms}$ each and an interstimulus interval of $30 \mathrm{~ms}$.

The occurrence of $\mathrm{T} 1$ in the stimulus stream was varied randomly between positions 7,8 , and 9 to reduce the predictability of first target onset. T2 was presented directly after T1 (lag 1) or after another 2, 4, or 7 distractors (lags 3, 5, and 8, respectively) (see Fig. 1). Both targets were to be reported (order of report was not considered) after the RSVP (the 

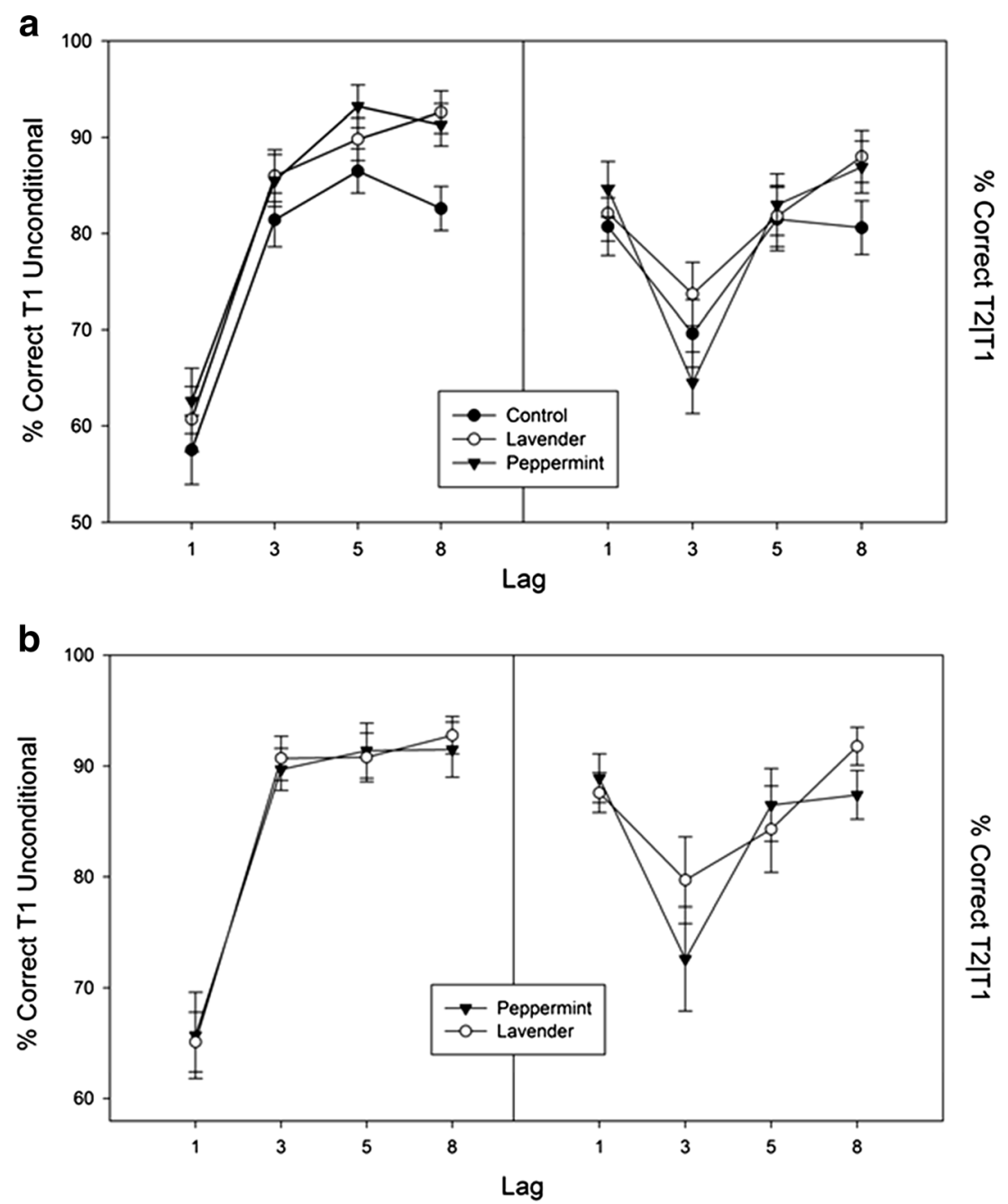

Fig. 1 a. Experiment 1: T1 (unconditional) performance (left panel) and $\mathrm{T} 2$ performance given $\mathrm{T} 1$ correct (T2|T1) (right panel), as a function of lag and aroma group. b. Experiment 2: T1 (unconditional) performance

question being "which two targets did you see?") by pressing the corresponding digit keys. A full experimental session lasted $10 \mathrm{~min}$ and contained one block of 144 trials (3 locations of $\mathrm{T} 1 \times 4$ lags $\times 12$ repetitions).

\section{Procedure and design}

Upon arrival at the laboratory, participants were asked to rate their mood on a $9 \times 9$ pleasure $\times$ arousal grid (Russell, Weis, $\&$ Mendelsohn, 1989), with values ranging from -4 to 4 . Afterward, participants were asked to perform the RSVP task (both the training and the experimental blocks) in either a peppermintor a lavender-scented testing room. The testing room was the same for all participants, and they all underwent the exact same procedure. Peppermint and lavender odors were selected given their opposite effects on attentional state (Barker et al., 2003; Basevitch et al., 2011; Ho \& Spence, 2005; Ilmberger et al., 2001; Moss et al., 2003). "De Tuinen" pure essential oils (De Tuinen Aromatherapie) of peppermint and lavender were used to (left panel) and T2 performance given T1 correct (T2|T1) (right panel), as a function of lag and aroma session

generate the ambient aromas. Following Sellaro et al. (2014, under revision), four drops of the appropriate oil were applied to a candle diffuser, diluted in $30 \mathrm{ml}$ of water. Two separate diffusers were used for spreading the two aromas. The diffuser was out of participants' sight, and the candle was switched on $20 \mathrm{~min}$ before the testing session started to ensure a uniform diffusion in the testing room. After completion of the RSVP task, participants again rated their mood. In Experiment 1, participants served in a single session with one aroma. In Experiment 2, participants took part in two experimental sessions (peppermint vs. lavender), separated by 3-7 days. The order of the two aroma conditions was counterbalanced across participants.

\section{Statistical analysis}

For both Experiments 1 and 2, an a priori power analysis was performed to estimate the approximate number of participants required for detecting medium effect sizes $(\approx .40)$, given the traditional .05 criterion of statistical significance. The 
calculation was made using $\mathrm{G}^{*}$ Power 3.1.7 program (Faul, Erdfelder, Lang, \& Buchner, 2007).

A significance level of $p<.05$ was adopted for all tests. T1 and T2 accuracy data were submitted to repeated measures analyses of variance (ANOVAs) with lag $(1,3,5$, and 8$)$ as a within-subjects variable and aroma condition (lavender vs. peppermint vs. control) as a between-subjects (group) factor (in Experiment 1) or within-subjects factor (lavender vs. peppermint; Experiment 2). T2 accuracy was based only on those trials on which T1 was correctly reported (T2|T1). Mood was analyzed separately by means of ANOVAs with effect of time (first vs. second) as a within-subjects factor and aroma condition as a between-subjects (group) factor (in Experiment 1) or a within-subjects factor (Experiment 2).

\section{Experiment 1}

\section{Participants}

Sixty-six healthy young adults, naïve about the purpose of the experiment, with a mean age of 20.7 years $(S D=2.4$, range 18-29), participated for partial fulfillment of course credit or a financial reward. Participants were equally distributed in three experimental groups. Twenty-two participants $(2$ male, 20 female) were exposed to lavender aroma, 22 (2 male, 20 female) to peppermint aroma, and 22 ( 3 males, 19 female) to no aroma. Two participants in the control group were excluded because of technical problems with the internal clock of EPrime. Written informed consent was obtained from all participants after a detailed explanation of the study procedures. The protocol was approved by the local ethical committee (Leiden University, Faculty of Social and Behavioral Sciences).

\section{Results}

The T1 ANOVA showed a significant lag effect, $F(3,183)=$ $181.60, p<.0001, \eta_{\mathrm{p}}^{2}=.75$. As Fig. 1 indicates, this effect was due to a dip in performance at lag 1 -that is, when T2 immediately followed $\mathrm{T} 1$. This pattern is typical if $\mathrm{T} 1$ and $\mathrm{T} 2$ belong to the same category (e.g., digits) and meet the same selection criteria and when the presentation rate is fast. These conditions are thought to increase the competition between $\mathrm{T} 1$ and $\mathrm{T} 2$ representations if they occur closely in time, with $\mathrm{T} 2$ outperforming T1 more often than at longer lags (Colzato et al., 2007; Hommel \& Akyürek, 2005; Potter, Staub, \& O'Connor, 2002). The aroma group effect was not significant, $F(3,183)=2.15, p=.13, \eta_{\mathrm{p}}^{2}=.07$, nor was the interaction between group and lag, $F(3,183)=0.97, p=.45, \eta_{\mathrm{p}}^{2}=.03$.

The T2 ANOVA revealed a significant lag effect, $F(3,183)=$ $49.70, p<.0001, \eta_{\mathrm{p}}^{2}=.45$, indicating a marked $\mathrm{AB}$ with good performance at lag 1 (lag 1 sparing; Visser, Bischof, \& Di Lollo, 1999), a considerable dip at lags 3 and 5, and recovered performance at lag 8 (see Fig. 1). As was predicted, aroma affected the magnitude of the $\mathrm{AB}$, as indicated by an interaction between group and lag, $F(6,183)=3.42, p<.01, \eta_{\mathrm{p}}^{2}=.10$. Post hoc multiple comparison tests (Fisher LSD) revealed that the peppermint group showed a greater $\mathrm{AB}$ than the lavender group $(p=.043)$ and the control group $(p=.006)$, while no difference in $\mathrm{AB}$ size was observed between the control group and the lavender group $(p=.41)$. The main effect of group was not significant, $F<1, p=.69$.

The mood ANOVA showed that participants tended to experience less arousal at the second than at the first measurement $(0.5$ vs. 0.1$), F(1,61)=7.42, p=.054, \eta_{\mathrm{p}}^{2}=.11$, whereas pleasure levels were constant across the two measurements $(0.8$ vs. 0.7$), F(1,61)=2.06, p=.16, \eta_{\mathrm{p}}^{2}=.03$. Importantly, for both arousal ( 0.4 vs. 0.0 and 0.8 vs. 0.4 for lavender and peppermint, respectively) and pleasure ( 0.8 vs. 0.5 and 0.6 vs. 0.7 for lavender and peppermint, respectively) analyses, neither the main effect of group nor the interaction between group and time reached the significance levels, $F_{\mathrm{s}}<1.1, p_{\mathrm{s}} \geq .34$. This suggests that we can rule out an account of our results in terms of (conscious) mood and arousal changes.

\section{Experiment 2}

\section{Participants}

Twenty participants ( 4 male, 16 female), naïve about the purpose of the experiment, who did not take part in Experiment 1, underwent the two testing sessions. Written informed consent was obtained from all participants; the protocol was approved by the local ethical committee (Leiden University, Institute for Psychological Research).

\section{Results}

The T1 ANOVA showed a significant lag effect, $F(3,57)=$ $77.86, p<.0001, \eta_{\mathrm{p}}^{2}=.80$. As in Experiment 1, this effect was due to a dip in performance at lag 1 -that is, when T2 immediately followed T1 (see Fig. 1). The main effect of aroma was not significant, nor was the interaction, $F_{\mathrm{s}}<1$, $p_{\mathrm{s}} \geq .87$.

The T2 ANOVA revealed a significant lag effect, $F(3,57)=$ 11.44, $p<.0001, \eta_{\mathrm{p}}^{2} .38$, indicating the typical $\mathrm{AB}$ pattern: good performance at lag 1 , a considerable dip at lags 3 and 5, and recovered performance at lag 8 (see Fig. 1). As was predicted, and in agreement with Experiment 1, aromas modulated the magnitude of the $\mathrm{AB}$, as indicated by a two-way interaction between session and lag, $F(3,57)=4.99, p<.005$, $\eta_{\mathrm{p}}^{2}=.21$ : In the peppermint session, participants showed a 
deeper $\mathrm{AB}$ than in the lavender session. The main effect of aroma was not significant, $F=1.46, p=.21$.

The mood ANOVA performed on arousal data ( 0.6 vs. 0.0 and 0.6 vs. -0.1 for lavender and peppermint, respectively) and on mood data (1.3 vs. 0.8 and 0.9 vs. 1.1 for lavender and peppermint, respectively) revealed no main effect of aroma, $F_{\mathrm{s}}<1, p_{\mathrm{s}} \geq .88$, or time, $F_{\mathrm{s}}<2.9, p_{\mathrm{s}} \geq .10$, nor an interaction, $F_{\mathrm{s}}<1.47, p_{\mathrm{s}} \geq .24$. This suggests that, again, we can rule out an account of our results in terms of (conscious) mood and arousal changes.

\section{Conclusions}

Our aim was to investigate whether being exposed to either a stimulating aroma (i.e., peppermint) or a relaxing aroma (i.e., lavender) changes the way people allocate their attention across time, as revealed by the $\mathrm{AB}$ task. As was expected, the $\mathrm{AB}$ was less pronounced when participants were exposed to the more relaxing aroma, suggesting that aromas can act as a cognitive modulator, supporting either a temporally focused (i.e., peppermint) or a temporally distributed (i.e., lavender) attentional state. This would account for the significantly smaller $\mathrm{AB}$ in the lavender session, which can be taken to reflect a relaxed, less focused attitude toward the task, as in Olivers and Nieuwenhuis's (2005) study, where reduced ABs were observed when participants engaged in distracting mental activity, such as free-associating on a task-irrelevant theme or listening to music (see footnote 1).

Even though our study did not include neurophysiological measures and, thus, cannot directly inform about the neural underpinnings of our effects, our observations fit with the outcomes of electrophysiological studies of the $\mathrm{AB}$ and of aroma effects. For one, it has been shown that exposure to lavender increases cortical beta power, accompanied by drowsiness (Diego et al., 1998; Field et al., 2005; Lorig, Herman, Schwartz, \& Cain, 1990). For another, Gross et al. (2004) found that increased long-range phase synchronization in the beta band was associated with a less pronounced $\mathrm{AB}$. Taken together, these findings would fit with our hypothesis that the relaxing lavender odor induces a more temporally distributed attentional state that, as it would work against attentional overinvestment, reduces the $\mathrm{AB}$, as compared with the stimulating peppermint aroma.

It is interesting to note that we did not find any evidence that mood or arousal changes might directly be responsible for the observed effects. However, our measures relied on conscious self-assessment and, thus, reflect merely conscious aspects of mood and arousal. This does not allow us to exclude the possible impact of more implicit mood and arousal changes that future studies might consider by including physiological measurements, such as galvanic skin response, heart rate, and diastolic and systolic blood pressure.
Taken altogether, these results show that performance in an $\mathrm{AB}$ task is not sufficiently accounted for by the assumption of a structural bottleneck. Apparently, the allocation of attention in time and the temporal dynamics of attention are determined not only by task instructions and related circumstances (Akyürek et al., 2007; Di Lollo et al., 2005; Gross et al., 2006; Olivers \& Nieuwenhuis, 2005; Shapiro et al., 2006) but also by environmental factors, at least if, and to the degree, that they induce a more focused or more distributed state of mind.

\section{References}

Akyürek, E. G., Riddell, P. M., Toffanin, P., \& Hommel, B. (2007). Adaptive control of event integration: Evidence from event-related potentials. Psychophysiology, 44, 383-391.

Barker, S., Grayhem, P., Koon, J., Perkins, J., Whalen, A., \& Raudenbush, B. (2003). Improved performance on clerical tasks associated with administration of peppermint odor. Perceptual and Motor Skills, 97, 1007-1010.

Basevitch, I., Thompson, B., Braun, R., Razon, S., Arsal. G., Tokac, U., Filho, E., Nacimiento-Razor, T., \& Tenenbaum, G. (2011). Olfactory effects on attention allocation and perception of exertion. The Sport Psychologist, 25, 144-158.

Chun, M. M., \& Potter, M. C. (1995). A two-stage model for multiple target detection in rapid serial visual presentation. Journal of Experimental Psychology: Human Perception and Performance, 21, 109-127.

Colzato, L. S., Bajo, M. T., van den Wildenberg, W., Paolieri, D., Nieuwenhuis, S. T., La Heij, W., \& Hommel, B. (2008a). How does bilingualism improve executive control? A comparison of active and reactive inhibition mechanisms. Journal of Experimental Psychology: Learning, Memory, and Cognition, 34, 302-312.

Colzato, L. S., Slagter, H., de Rover, M., \& Hommel, B. (2011). Dopamine and the management of attentional resources: Genetic markers of striatal D2 dopamine predict individual differences in the Attentional Blink. Journal of Cognitive Neuroscience, 23, 3576-3585.

Colzato, L. S., Slagter, H. A., Spapè, M., \& Hommel, B. (2008b). Blinks of the eye predict blinks of the mind. Neuropsychologia, 46, 31793183 .

Colzato, L. S., Spapè, M., Pannebakker, M., \& Hommel, B. (2007). Working memory and the Attentional Blink: Blink size is predicted by individual differences in operation span. Psychonomic Bulletin \& Review, 14, 1051-1057.

Dale, G., \& Arnell, K. M. (2010). Individual differences in dispositional focus of attention predict attentional blink magnitude. Attention, Perception, \& Psychophysics, 72, 602-606.

Di Lollo, V., Kawahara, J. I., Ghorashi, S. M., \& Enns, J. T. (2005). The attentional blink: Resource depletion or temporary loss of control? Psychological Research, 69, 191-200.

Diego, M.A., Jones, N.A., Field, T., Hernadez-Reif, M., Schanberg, S., Kuhn, C., ... Galamaga, M. (1998). Aromatherapy positively affects mood, EEG, patterns of alertness and math computations. International Journal of Neuroscience, 96, 217-224.

Dux, P. E., \& Marois, R. (2009). The attentional blink: A review of data and theory. Attention, Perception, \& Psychophysics, 71, 1683-1700.

Faul, F., Erdfelder, E., Lang, A.-G., \& Buchner, A. (2007). G*Power 3: A flexible statistical power analysis for the social, behavioral, and biomedical sciences. Behavior Research Methods, 39, 175-191. 
Field, T., Diego, M., Hernandez-Reif, M., Cisneros, W., Feijo, L., Vera, Y., ... He, Q. (2005). Lavender fragrance cleansing gel effects on relaxation. International Journal of Neuroscience, 115, 207-222.

Gross, J., Schmitz, F., Schnitzler, I., Kessler, K., Shapiro, K., Hommel, B., \& Schnitzler, A. (2004). Long-range neural synchrony predicts temporal limitations of visual attention in humans. Proceedings of the National Academy of Sciences USA, 101, 13050-13055.

Gross, J., Schmitz, F., Schnitzler, I., Kessler, K., Shapiro, K., Hommel, B., $\&$ Schnitzler, A. (2006). Anticipatory control of long-range phase synchronization. European Journal of Neuroscience, 24, 2057-2060.

Hertz, S. R. (2009). Aromatherapy facts and fictions: a scientific analysis of olfactory effects on mood, physiology and behavior. International Journal of Neuroscience, 119, 263-290.

Ho, C., \& Spence, C. (2005). Olfactory facilitation of dual-task performance. Neuroscience Letters, 389, 35-40.

Hommel, B., \& Akyürek, E. (2005). Lag-1 Sparing in the attentional blink: benefits and costs of integrating two events into a single episode. Quarterly Journal of Experimental Psychology, 58A, $1415-1433$.

Ilmberger, J., Heuberger, E., Mahrhofer, C., Dessovic, H., Kowarik, D., \& Buchbauer, G. (2001). The influence of essential oils on human attention. I: Alertness. Chemical Senses, 26, 239-245.

Johnson, A. J. (2011). Cognitive facilitation following intentional odor exposure. Sensors, 11, 5469-5488.

Jolicoeur, P., \& Dell'Acqua, R. (1998). The demonstration of short-term consolidation. Cognitive Psychology, 36, 138-202.

Lorig, T. S., Herman, K. B., Schwartz, G. E., \& Cain, W. S. (1990). EEG activity during administration of low concentration odors. Bulletin of the Psychonomic Society, 28, 405-408.

Martens, S., \& Johnson, A. (2005). Timing attention: Cuing target onset interval attenuates the attentional blink. Memory \& Cognition, $33(2), 234-240$.

Martens, S., Munneke, J., Smid, H., \& Johnson, A. (2006). Quick minds don't blink: Electrophysiological correlates of individual differences in attentional selection. Journal of Cognitive Neuroscience, 18(9), $1423-1438$
Martens, S., \& Valchev, N. (2009). Individual differences in the attentional blink: The important role of irrelevant information. Experimental Psychology, 56(1), 18-26.

Moss, M., Cook, J., Wesnes, K., \& Duckett, P. (2003). Aromas of rosemary and lavender essential oils differentially affect cognition and mood in healthy adults. International Journal of Neuroscience, 113, 15-38.

Olivers, C. N. L., \& Nieuwenhuis, S. (2005). The beneficial effect of concurrent task-irrelevant mental activity on temporal attention. Psychological Science, 16, 265-269.

Olivers, C. N. L., \& Nieuwenhuis, S. (2006). The beneficial effects of additional task load, positive affect, and instruction on the attentional blink. Journal of Experimental Psychology: Human Perception and Performance, 32, 364-379.

Potter, M. C., Staub, A., \& O'Connor, D. H. (2002). The time course of competition for attention: attention is initially labile. Journal of Experimental Psychology: Human Perception and Performance $28,1149-1162$.

Raymond, J. E., Shapiro, K. L., \& Arnell, K. M. (1992). Temporary suppression of visual processing in an RSVP task: An attentional blink? Journal of Experimental Psychology: Human Perception and Performance, 18, 849-860.

Russell, J. A., Weis, A., \& Mendelsohn, G. A. (1989). Affect grid: A single-item scale of pleasure and arousal. Journal of Personality and Social Psychology, 57, 493-502.

Shapiro, K., Schmitz, F., Martens, S., Hommel, B., \& Schnitzler, A. (2006). Resource sharing in the attentional blink. Neuroreport, 17, 163-166.

Taatgen, N. A., Juvina, I., Schipper, M., Borst, J., \& Martens, S. (2009). Too much control can hurt: A threaded cognition model of the attentional blink. Cognitive Psychology, 59, 1-29.

Visser, T., Bischof, W. F., \& Di Lollo, V. (1999). Attentional switching in spatial and non-spatial domains: evidence from the attentional blink. Psychological Bulletin, 125, 458-469.

Vogel, E. K., Luck, S. J., \& Shapiro, K. L. (1998). Electrophysiological evidence for a postperceptual locus of suppression during the attentional blink. Journal of Experimental Psychology: Human Perception and Performance, 24, 1656-1674. 